\title{
Key Technologies of the Independent and Innovative Communications-Based Train Control Systems in China
}

\author{
Bo Liu ${ }^{1} \cdot$ Chunhai Gao ${ }^{1}$
}

Received: 9 November 2015/Revised: 24 March 2016/ Accepted: 27 March 2016/Published online: 16 April 2016

(C) The Author(s) 2016. This article is published with open access at Springerlink.com

\begin{abstract}
In the past 10 years, the independent and innovative communications-based train control system has experienced breakthroughs in key technologies by conducting pilot experiments, field tests, and demonstration projects. For the first time, the design theories and technical aspects of moving block systems, which cover the entire life cycle of fail-safe systems, are set up. The models and algorithms are proposed to control the safe operation of trains. The safety technologies for short-interval train sequencing are adopted to realize the $90 \mathrm{~s}$ headway between consecutive trains. The optimized automatic train operation strategies are used to save energy and allow trains to safely reach their destinations. The highly dependable and bi-directional train-to-ground communication technologies are introduced for reliable transmission of safety related train-to-ground information in a complex environment which is compatible with multiple media, such as free waves, leaky waveguides, and leaky feeders. The simulation, tests, and verification technologies based on the minimum system and test cases were adopted to conduct the following: principle demonstration, functional tests, integrated tests, field failure data replay, analysis and disposal, system upgrade, and maintenance.
\end{abstract}

Keywords Communications-based train control (CBTC) - Moving block - Automatic train operation (ATO) - Safe train operation

Bo Liu

18811446007@163.com

Chunhai Gao

jun.chen@bj-tct.com

1 Beijing Traffic Control Technology CO, Ltd, Beijing, China

Editor: Xuesong Zhou

\section{Background}

Communications-based train control (CBTC) is the most advanced technology in the field of urban rail traffic signal control. Since 2004, CBTC has been adopted in newly constructed and upgraded old subway lines in Beijing, Shanghai, Guangzhou, and other cities [1]. China's first set of CBTC systems with completely independent intellectual property rights was implemented in the Yizhuang and Changping lines of the Beijing subway on December 30, 2010. The Yizhuang line is currently the most complete line in terms of functionality, and had the best performance in the world at the time of its opening. Based on the primary evaluation, an additional $6000-\mathrm{km}$ line will be built into China's subway systems by 2020 . The implementation of CBTC systems made China the fourth Country, after Germany, France, and Canada, to own the key technologies and to successfully apply them in practical engineering practices. The successful opening and operation of the Yizhuang line is an indication that the independent research, development, and engineering application of the domestic signal system have made significant progress [2].

The development of self-dependent innovative CBTC systems proposed in this paper takes 10 years and can be divided into five stages as follows [3]:

- The first stage: professors and scholars put forward the scientific problem and conduct theoretical analysis.

- The second stage: universities complete research on key technologies.

- The third phase: operation users conduct field and pilot tests.

- The fourth stage: construction users develop demonstration projects. 
- The fifth stage: enterprises lead the application of research results to real-world implementation.

Our CBTC system can achieve the same design intervals and safety integrity levels (SIL) as those of the foreign signal companies that are experienced in signal systems development. However, the cost is more than $20 \%$ lower than other companies. Table 1 shows comparison between domestic and foreign CBTC systems.

As seen in the table above, except CBTC system supplied by HITACHI, our CBTC system as well as other foreign CBTC systems can achieve the design intervals that is within $90 \mathrm{~s}$. Meanwhile, our CBTC system follows the safety standard which is obeyed by most foreign CBTC systems and also is the highest standard in the industry.

\section{Key Technologies}

\subsection{System Design and Equipment Development}

The developed design theories and technologies of moving block with high security and high reliability cover the entire life cycle. We studied the rail transit CBTC system integration technology and method. According to the overall goal of the system and operation requirements, we use integrated method to combine the system-related discipline theory, method, technology, and demand, in turn, making an overall analysis of structure, environment, and function of the system.

\section{System design for moving block}

The moving block system design theory was established to realize the security tracking of the train group in a "single file" style and $90 \mathrm{~s}$ minimum interval [4]. It includes multiparticle models for train traction and braking, precise positioning model for multi-sensor fusion of single-train complex line, minimum distance information searching, early warning model, automatic collaborative energy-saving control model for train groups, strategies, and methods for bi-directional transmission of security control information between large-scale train groups and stations, etc.
2. Critical life-cycle safety system design

According to CEN-ELEC international standards, the system design and development process, which covers the entire life cycle, must be based on risk analysis and be able to trace risks back [5]. After the process was established, CBTC risk source identification component was completed. A reliability and security analysis model of a security module with interval and speed control was built. Nearly 100,000 test cases were done in the comprehensive test platform.

3. A secure computer platform investigated and developed independently

Based on the theory of fault tolerant computers, a fault tolerant and safety management (FTSM) mechanism was proposed. This mechanism can prevent known or unknown software and hardware faults. Design principles of space diversity and the time diversity (time triggered software scheduling mechanism) were adopted to meet the characteristics of deterministic fault security. Figure 1 shows a security framework adopted in Yichuan Line of the Beijing subway.

As seen in Fig. 1, this security framework is hierarchical. The layer of software is divided into three parts, application software, security platform software, and realtime multitasking operating system from top to down. There comes the layer of hardware. It contains double FTSM hardware, real-time multitasking operating system, and FTSM communication bus. On the bottom, dual redundancy high-speed-switched Ethernet is used to connect hardware.

\section{Technical equipment}

Technical equipments are developed based on integrated design, 2-level scheduling model, and three types of control. Research on equipment development with rank-free conversion and cooperative control was completed. The integrated design, which includes multiple components ATS (Automatic Train Supervision), ATP (Automatic Train Protection), ATO (Automatic Train Operation), and CI (Computer Interlocking), was proposed. Then the
Table 1 Comparison of domestic and foreign CBTC

\begin{tabular}{llll}
\hline Signal system & Supplier & Design interval & Safety standards \\
\hline Introduced CBTC & THALES & Within $90 \mathrm{~s}$ & SIL4, highest standard \\
& SIEMENS & Within $90 \mathrm{~s}$ & SIL4, highest standard \\
& ALSTOM & Within $90 \mathrm{~s}$ & SIL4, highest standard \\
& ANSALDO & Within $90 \mathrm{~s}$ & SIL4, highest standard \\
& Bombardier & Within $90 \mathrm{~s}$ & SIL4, highest standard \\
& HITACHI & $120 \mathrm{~s}$ & Japanese standard \\
Independent and innovative CBTC & TCT & Within $90 \mathrm{~s}$ & SIL4, highest standard \\
\hline
\end{tabular}


Fig. 1 Security framework of Yizhuang Line of Beijing Subway
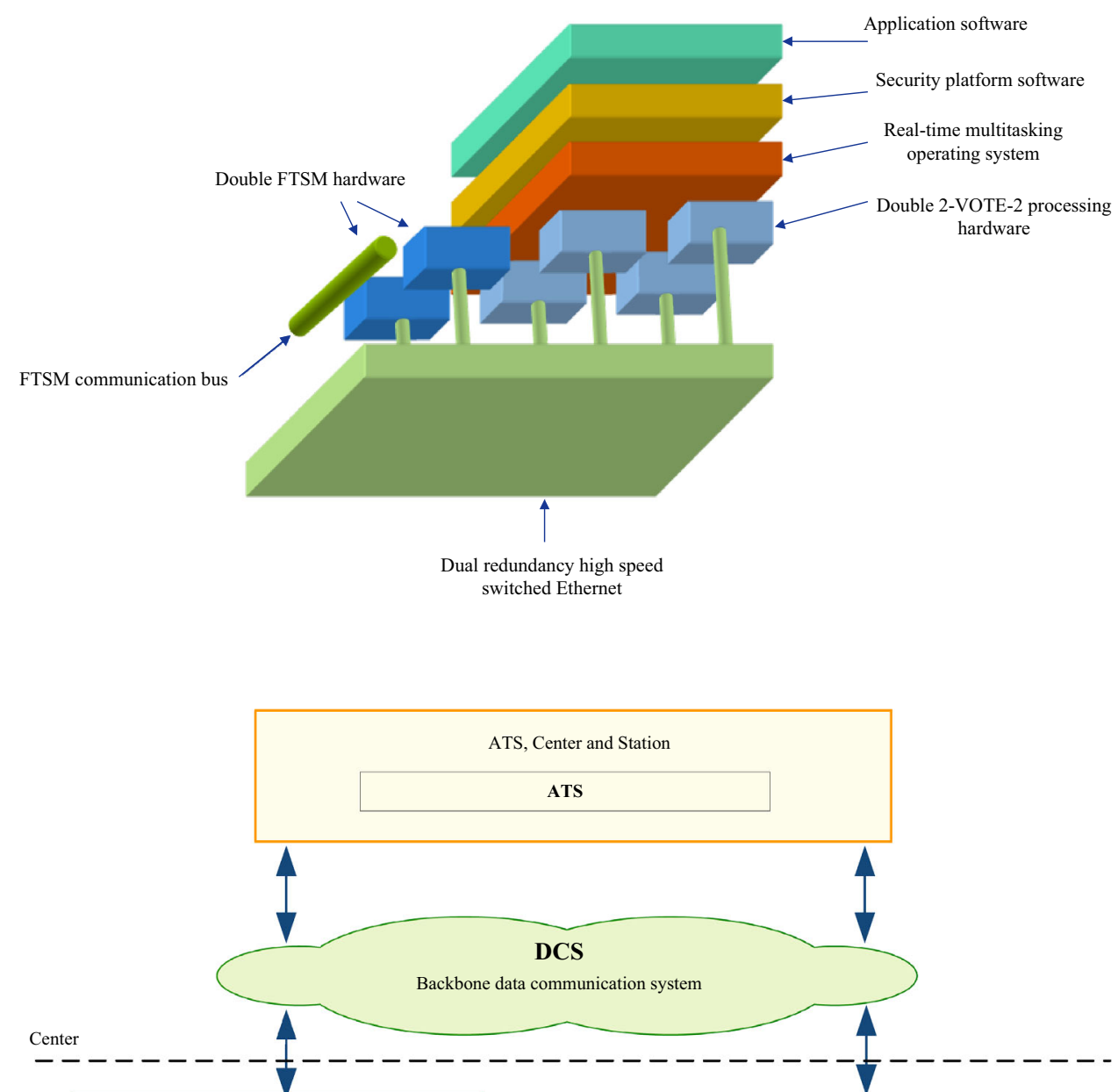

Fig. 2 Integrated design, 2-level scheduling model, and 3 types of control complete set of technical equipment was developed. The complex coordination of 3-level asynchronous, non-intrusive, multi-vehicle-mixed operations of the mobile block, fixed block, and the interlocking of the station was constructed (Fig. 2).
As seen in Fig. 2, ATS is on the top level. It is always put in the command center and stations. Its main function is to monitor the train running state, use the software to realize the automatic networking, communications, and train operation management. There comes the DCS (Backbone 
data communication system) [6]. It takes the responsibility of data transmission between ATS and other blocks below. CI plays a role in controlling and monitoring the fixed block which is composed of annunciators, turnouts, axle counters platform equipment. ATP's main function is to achieve the train interval control and speed control, and then ensure driving safety. In order to do this, there is a channel for the information transmission between the on-board ATP equipment and ground ATP equipment, which we called Train-to-ground wireless communication system here. ATO in the ground and ATO in the train work together to fulfill automatic driving between stations, speed adjustment, and precise stop inside the stations.

\subsection{Safety Protection and Automatic Driving}

The safety protection technology for highly dense train traffic, which connects the vehicle with the ground, was proposed initially at the domestic level. The problems of unmanned operation and safety tracing for high speed, high density, complex lines, and adverse weather conditions were addressed. This ensures the safety of the train operations around the clock. Optimal automatic driving technology was created for the first time at the domestic level. Automatic adjustment of operation time tables in the complex environment of multi-objective optimization was used, and the adjustment error is less than $5 \mathrm{~s}$. Energysaving optimization control of complex lines was realized [7]. Precise stopping of trains at stations was achieved, and the parking precision was less than $0.3 \mathrm{~m}$. A few key achievements are listed below:

1. Optimal automatic driving technology was adopted.

2. Train dynamics model, which combines single- and multi-particle models and then realizes train overspeed protection control, was established (Fig. 3). If the speed of train reaches to $\mathrm{v}_{4}$, it strikes the common braking line, so the system will activate the common

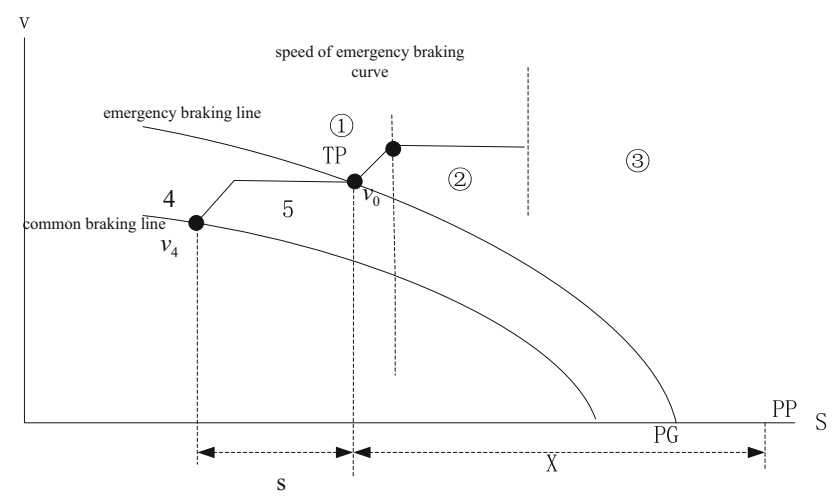

Fig. 3 Train dynamics model which combines simple particles and multi particles braking model. Seriously, if the speed reaches to $\mathrm{v}_{0}$, it strikes the emergency braking line, so the system will activate the emergency braking model to cut down the speed.

3. The train group safety control strategy was adopted.

4. Optimal automatic driving technology was applied.

\subsection{Bi-directional Large Capacity Transmission Between the Vehicle and Ground}

Three transmission modes, namely free wireless wave, leaky waveguide, and leaky feeder, were adopted for the first time in the self-dependent innovative CBTC system. The train operation control information realizes bi-directional transmission. As a result, the engineering adaptability and flexibility of the CBTC system are greatly improved. The information transmission synchronization correction algorithm was proposed. CBTC security transfer protocol was developed. The CBTC information redundancy transmission network was developed independently, providing a reliable and safe data channel between each subsystem of the CBTC system. We studied all the way of WLAN that are used for railway traffic control system at present, including IEEE802.11, IEEE802.11b. IEEE802.11g [8]. Meanwhile, we also studied the leaky waveguide. Finally, we made experiments of IEEE802.11g train-ground transmission mode in the field.

\subsection{Simulation, Testing, and Verification}

1. Technology of a semi-physical system with the hardware in the loop

Based on the event-driven technology, the CBTC simulation and testing platform was developed [9]. The minimum system with minimization hardware and maximization function was established. Based on visual identification characteristics of rail transit virtual intelligent scene of generation technology, flexible virtual reality, and the mutual control were proposed, and the comprehensive test of visual and high coverage was operated.

2. Test sequence based on fault injection and script verification

Using a formal method, the non-formal description of test specification was described and the test sequence was optimized [10]. The test case sets of the CBTC system was formed using data-driven testing technologies. The test sequence toolset based on engineering data was set up through the method of hierarchical decision tree analysis. Subsequently, test sequences under different fault scenarios were generated automatically. 
3. Recording, playback, and evaluation technologies

Logging efficiency and capacity were improved greatly by $\log$ record mode based on encoding storage. The recorded data, which uses log format standardization, can be played again in the test platform, providing the basis for the dynamic analysis of the operation status of equipment evaluation and replication site problems [11].

\subsection{Other Innovative Results}

In the process of the implementation of the CBTC system, the structure of the signal system was optimized and functional limitation was surpassed by large numbers of research and in-depth analysis of the international standards and systems [12]. The innovative results are as follows: the standard system equipment configuration principles, signal equipment layout design principles, design principles of CBTC backup mode, platform screen door linkage function of intermittent type, interface design principles, design and on-site verification of signal system with 2-min tracking interval running ability, the guide tube optimization scheme, wireless network security testing, and driving process fault handling function optimization [13-15].

\section{Conclusion}

Independent innovation of the CBTC system can encourage us to keep up with the latest technological development of the international train operation control systems. Core technologies of the CBTC system, such as bi-directional transmission technology between the vehicle and ground, precise positioning of the train, automatic safety protection, and automatic driving, are mastered [16]. CBTC for urban rail transit systems in China were developed in full accordance with the CEN-ELEC international security standards, thereby reaching the highest level internationally.

The successful application of independent innovation CBTC systems breaks the long-term monopoly of the core technology of the subway signal in our country, bringing significant economic and social benefits. This innovation brings down the price of imported system by $20 \%$ and reduces the cost of our own CBTC by $10 \%$. With the development of the core technologies and industrial chain, the independent CBTC system will be the most cost-effective system, providing a solid technical foundation for the construction and operation of urban rail transit in China.

Acknowledgments This work is supported by the Beijing municipal science and technology plan project (Project Number: D15110000581 5001) and the Beijing urban rail operation control system engineering technology research center.
Open Access This article is distributed under the terms of the Creative Commons Attribution 4.0 International License (http://crea tivecommons.org/licenses/by/4.0/), which permits unrestricted use, distribution, and reproduction in any medium, provided you give appropriate credit to the original author(s) and the source, provide a link to the Creative Commons license, and indicate if changes were made.

\section{References}

1. Fraszczyk A, Brown P, Duan S (2015) Public perception of driverless trains. Urban Rail Transit 1(2):78-86

2. Batty P, Palacin R (2015) The circumvention of barriers to urban rail energy efficiency. Urban Rail Transit 1(2):71-77. doi:10. 1007/s40864-015-0014-9

3. An J, Yang D, Kang Q, Wang L, Wu Q (2008) Security radio data transmission mode based on turbo codes for the CBTC system. Intelligent control and automation, 2008. WCICA 2008. 7th world congress on 25-27 June 2008, pp 6663-6668

4. Fitzmanrice M (2006) Use of $2.4 \mathrm{GHz}$ frequency band for communication based train control data communication system. In: Rail conference, 2006. Proceeding of the 2006 IEEE/ASME joint, 4-6 April 2006, pp 263-267

5. Gao C, Tang T, Yao F (2006) Research and design of vehicle equipment ATP based on CENELEC. J China Railw Soc 28(1):99-107

6. Tang T, Yao F, Gao C (2005) Research on security assessment and authentication system of rail traffic signal system. Urban Rapid Rail Transit 17(6):28-32

7. Huang Y, Tang T (2008) Research on virtual simulation technology in metro train operation simulation system. J Syst Simul 20(12):3208-3211

8. Li Z, Bin N (2010) Design of CBTC train-ground communication system based on $802.11 \mathrm{~g}$ IEEE standard. China Railw Sci 31(5):119-124

9. Ma L, Gao B (2004) Design and implementation of a high security and fault tolerant control computer. China Railw Sci 8

10. Wang H (2010) Analysis method and application of functional hazard analysis based on model. Trans Beijing Inst Technol 7:849-854

11. Pascoe RD, Eichorn TN (2009) What is communication-based train control? IEEE J Mag 4

12. Zhao X, Tang T, Yan F (2009) A functional safety analysis approach for analyzing CBTC system. In: IEEE conference publications, 2009, vol 3

13. Chen L, Ning B, Xu T-H (2007) Research on modeling and simulation of vehicle-on-board automatic train protection subsystem of communication based train control system. In: IEEE conference publications, 2007, vol 2

14. Schifers C, Hans G IEEE standard for communication-based train control (CBTC) performance and functional requirements

15. Peng Z, Li G, Wang H, Wu Q (2008) A wireless transmission mechanism of the wireless CBTC system and performance analysis. Audio, language and image processing, 2008. ICALIP 2008. International Conference on 7-9 July 2008, pp 443-448

16. Zhang Z, Wang CQ, Zhang W (2015) Status analysis and development suggestions on signaling system of Beijing rail transit. Urban Rail Transit 1(1):1-12 